\title{
Impacto de un programa cuerpo-mente basado en ejercicios de fisioterapia y técnicas psicológicas en el bienestar psíquico de una población de pacientes diabéticos tipo $2^{*}$
}

Impact of a Body Mind Program Base on Physiotherapeutic Exercises and Psychological Techniques in the Psychic Welfare of a Diabetics

Patients Type 2

Recepción: 17 Diciembre 2012 | Aprobación: 23 Enero 2017

\author{
María Orosia Lucha-López \\ Universidad de Zaragoza, España \\ ORCID: http://orcid.org/0000-0002-9930-3903 \\ Ana Carmen Lucha-López \\ Universidad de Zaragoza, España \\ José Miguel Tricás-Moreno \\ Universidad de Zaragoza, España \\ Carlos Salavera-Bordás \\ Universidad de Zaragoza, España \\ Elena Estébanez De Miguel \\ Universidad de Zaragoza, España \\ Concepción Vidal-Peracho \\ Universidad de Zaragoza, España
}

\footnotetext{
a Autor de correspondencia. Correo electrónico: orolucha@unizar.es
}

Para citar este artículo: Lucha-López, M. O., LuchaLópez, A. C., Tricás-Moreno, J. M., SalaveraBordás, C., Estébanez De Miguel, E., \& VidalPeracho, C. (2017). Impacto de un programa cuerpomente, basado en ejercicios de fisioterapia y técnicas psicológicas, en el bienestar psíquico de una población de pacientes diabéticos tipo 2. Universitas Psychologica, 16(1), 1-13. http://dx.doi.org/10.11144/Javeriana.ups y16-1.ipcb

\section{RESUMEN}

En los pacientes con diabetes tipo 2, el riesgo de sufrir algún tipo de malestar psicológico con síntomas de depresión y ansiedad, es aproximadamente el doble que en población normal. Cuando son síntomas relacionados con condiciones concomitantes, físicas y psicológicas deben ser tratados. El objetivo de este trabajo, fue examinar los efectos de un programa de ejercicios de fisioterapia y técnicas psicológicas, en el bienestar psíquico de una población diabética. El programa de 12 semanas se aplicó en 25 pacientes y se evaluaron: dolor, tensión arterial, antropometría, analítica sanguínea y bienestar psíquico (cuestionario de Goldberg de 28 ítems: GHQ-28). A pesar de la edad (84 $\%<$ de 65 años), solo el $12 \%$ trabajaban, debido a su situación clínica de alto riesgo cardiovascular, presencia de comorbilidades (96 \%) y dolor asociado. Todo ello afectaba negativamente el bienestar psíquico de los pacientes que puntuaron alto en el GHQ: disfunción social (14.8 \pm 2.2$)$, síntomas somáticos $(13.5 \pm 3.6)$, ansiedad e insomnio $(11.9 \pm 3.4)$ y depresión severa $(9.2 \pm 4.4)$. La situación clínica determinó el diseño del programa, que mostró gran eficacia terapéutica para el dolor y el aumento del bienestar psicológico, con gran repercusión en la puntuación total del GHQ, en los síntomas somáticos y en la disfunción social, aunque en la ansiedad e insomnio y la depresión los cambios fueron menores. En síntesis, el programa mejoró el bienestar físico y psíquico de la muestra, lo que aumenta la evidencia sobre la opción de incluir fisioterapia y 
psicología para tratar las circunstancias que causan malestar psíquico en la diabetes.

Palabras clave

diabetes mellitus; salud mental; modalidades de fisioterapia; técnicas psicológicas; terapia por ejercicio.

\begin{abstract}
Diabetic patients are around twice as likely to suffer from anxiety and depression symptoms as the general population. When these symptoms are related to concurrent conditions they should be treated. The objective of this study has been to examine the effects of a physical therapy exercises and psychological techniques program, in mental wellness of a diabetic population. The twelve weeks program was applied in 25 patients. Pain, arterial pressure, anthropometry, blood samples and mental wellness (with 28 items Goldberg questionnaire: GHQ-28) were evaluated. Though they were relatively young ( $84 \%$ $<$ than 65 years), only $12 \%$ were working, due to the high cardiovascular risk clinical situation, the existence of comorbidities (96\%) and the associated pain. These facts negatively affected the mental wellness of the people, so they scored high in GHQ questionnaire: social dysfunction (14.8 \pm 2.2$)$, somatic symptoms (13.5 \pm 3.6$)$, anxiety and insomnia $(11.9 \pm 3.4)$ and severe depression $(9.2 \pm$ 4.4). The clinical situation determined the design of the program, which showed good treatment effects for pain and general mental wellness, with high effect sizes, in GHQ total score, in somatic symptoms and in social dysfunction, though in anxiety and insomnia and severe depression the effect size was small. In conclusion, the program improved physical and psychological wellness of our patients. The evidence for inclusion of physical therapy and psychology techniques in the management of concurrent conditions in diabetes, source of mental discomfort, had been reinforced. Keywords

diaabetes mellitus; mental health; physical therapy modalities; phychological techniques; exercise therapy.
\end{abstract}

Recientes estudios muestran que en los pacientes con diabetes, el riesgo de sufrir algún tipo de malestar psicológico con síntomas de depresión y ansiedad es aproximadamente el doble que en la población normal (Buchberger et al., 2016; Tovilla-Zárate et al., 2012; Trento et al., 2012).

Estos síntomas no se deben relacionar directamente con el diagnóstico de depresión mayor, que requeriría, para ser establecido, el cumplimiento de una serie de criterios no examinados en la mayoría de los estudios. En ellos, el diagnóstico de este estado psíquico se realiza con cuestionarios autoadministrados que recogen la presencia de síntomas de malestar psíquico, si bien no están validados para el diagnóstico de trastorno depresivo mayor (Gonzalez, Fisher, \& Polonsky, 2011). Dichos síntomas parecen tener relación con otras condiciones y circunstancias de la diabetes; así, la ansiedad se asocia con dificultades en el autotratamiento de la enfermedad (Fisher et al., 2010) y la aparición de polineuropatía diabética periférica, los indicadores de severidad de la misma, los síntomas asociados y la repercusión de esta complicación en las actividades de la vida cotidiana ocasionan con el tiempo un incremento de los síntomas depresivos (Vileikyte et al., 2009). Además, las complicaciones de la diabetes, en general, se asocian tanto con síntomas de ansiedad como de depresión (Chien \& Lin, 2016; Tovilla-Zárate et al., 2012). Por todo ello, parecen existir factores relacionados con la enfermedad y sus repercusiones funcionales que contribuyen al desarrollo de malestar psíquico; estos factores deben ser tenidos en cuenta a la hora de plantear intervenciones que mejoren dicho malestar en estas poblaciones (González et al., 2011; Usán, Salavera, Murillo, \& Mejías, 2016; Vidal-Peracho et al., 2014).

La eficacia en el tratamiento sobre las repercusiones funcionales de la diabetes con técnicas fisioterápicas específicas ha sido observado y cuantificado mediante variables físicas (Adeniyi, Fasanmade, Sanya, \& Borodo, 2010; Lucha-López et al., 2012; Pariser, DeMeuro, Gillette, \& Stephen, 2010; Sartor et al., 2012), psíquicas (Liu, Miller, Burton, \& Brown, 2010) y sobre la calidad de vida (Nicolucci et al., 2012), con resultados muy prometedores.

Además, el estrés psicológico ha sido identificado como un factor precipitante de las complicaciones de la diabetes, debido a la activación de ciertos factores inflamatorios y a la alteración del sistema inmune (Hartmann et al., 2012), por lo que cada vez, una mayor cantidad de investigaciones programan intervenciones para la gestión del estrés en pacientes diabéticos (Lucha-López et al., 2014), con resultados positivos sobre cómo afrontar los síntomas de la 
enfermedad y mejorar el bienestar y la calidad de vida (Merkes, 2010).

Con este marco de referencia, se estableció como objetivo de este trabajo, examinar los efectos de un programa cuerpo-mente, basado en ejercicios de fisioterapia y técnicas psicológicas, en el bienestar psíquico de una población con diabetes tipo 2 .

\section{Método}

\section{Participantes}

El estudio se realizó con una muestra de 25 personas que asistían a consulta externa especializada en Endocrinología y Nutrición del Hospital Royo Villanova (Centro Médico de Especialidades Grande Covián) de la ciudad de Zaragoza. Se utilizó un procedimiento de muestreo consecutivo y de conveniencia, no probabilístico.

El protocolo del estudio fue aprobado por el Departamento de Fisiatría y Enfermería de la Universidad de Zaragoza y se realizó de acuerdo con los principios de la Declaración de Helsinki. Todos los participantes firmaron un consentimiento informado y se les comunicó que podían abandonar el estudio en cualquier momento y por cualquier motivo.

Se decidió no incluir grupo control, debido a que tanto la intervención con ejercicio terapéutico como con técnicas psicológicas han dado muestras de su eficacia y seguridad previamente, siguiendo las recomendaciones del punto 32 de la Declaración de Helsinki.

Los criterios de inclusión fueron: diagnóstico endocrinológico de diabetes mellitus tipo 2, índice de masa corporal (IMC) mayor o igual a 25 , edad entre 45 y 76 años, dieta y prescripción farmacológica similar a lo largo de todo el estudio.

\section{Instrumentos}

\section{Anamnesis}

Sexo, edad, edad de inicio de la diabetes, situación laboral, tipo de dieta en el último año, prescripción farmacológica, comorbilidades y escala analógica del dolor (VAS) en su forma horizontal (Telli \& Cavlak, 2006).

\section{Tensión arterial}

La medición de la tensión arterial sistólica (TAS) y diastólica (TAD) se realizó con un manguito adaptado a los pacientes obesos de acuerdo con el Seventh Report of the Joint National Committee on Prevention, Detection, Evaluation, and Treatment of High Blood Pressure ([JNC 7], 2004), tras un periodo de unos quince minutos en sedestación, con tensiómetro de reloj de un tubo (Riester Corporation).

\section{Antropometría}

Todos los datos se obtuvieron de acuerdo con el protocolo de evaluación de la International Society for the Advancement of Kinanthropometry (ISAK) (Norton et al., 1996). La altura se midió con estadiómetro de pared (Seca-Línea médica: estadiómetro desmontable, alcance $30-220 \mathrm{~cm}$ ), el peso con balanza electrónica (Biologica Company: TANITA TBF 300) y el perímetro de cintura con cinta métrica inextensible (TECSYMP/Instrumentos Médicos: cinta métrica de plástico 0-2 m).

\section{Analítica sanguínea}

La evaluación de los parámetros sanguíneos se llevó a cabo en el laboratorio del Centro Médico Grande Covián, mediante extracción sanguínea, tras un periodo de ayuno no inferior a 12 horas. Se analizaron los siguientes parámetros:

El colesterol total, las lipropoteínas de alta densidad (HDL-c), las lipoproteínas de 
baja densidad (LDL-c), los triglicéridos y el fibrinógeno, determinados por nefelometría (DADE-Behring Corporation). La glucemia, hemoglobina glicosilada (HbA1c) y Proteína C Reactiva Ultrasensible (PCR US) fueron cuantificadas mediante un analizador selectivo Modular (Roche Corporation). La Entidad Nacional de Acreditación (ENAC) validó los controles de calidad del laboratorio (número de acreditación: 742/LE1586).

\section{Cuestionario de Salud General de Goldberg}

Para evaluar el grado de malestar psíquico se empleó el Cuestionario de Salud General de Goldberg, General Health Questionnaire, de 28 items (GHQ-28). Es un instrumento para evaluar salud autopercibida, especialmente en lo que se refiere a la presencia de ciertos estados emocionales, en contextos como la atención primaria o en población general (Goldberg \& Williams, 1988). El GHQ-28 fue diseñado para ser de fácil aplicación, aceptable para los encuestados, relativamente corto y ha sido validado en población española (Lobo, PérezEcheverría \& Artal, 1986) y en población española con trastornos endocrinos (Lobo, PérezEcheverría, Jiménez-Aznárez, \& Sancho, 1988).

Este cuestionario evalúa los cambios de la función normal, más que los rasgos presentes a lo largo de toda la vida. Mide la incapacidad para seguir llevando a cabo las funciones "saludables" normales y la aparición de nuevos fenómenos de malestar psíquico. La alteración de las funciones saludables tendría relación con, por ejemplo, ser incapaz de dormir debido a preocupaciones, sentirse bajo presión, ser incapaz de concentrarse y sentirse incapaz de enfrentarse a los problemas propios. El malestar podría concretarse en la existencia de trastornos distímicos (los de menor entidad en la gravedad de los trastornos psíquicos), así como en síntomas somáticos menores.

La puntuación del cuestionario determina la posición de un individuo en la dimensión hipotética subyacente del malestar psíquico, en el eje que va desde la normalidad hasta un claro trastorno. El GHQ-28, además de la puntuación total, muestra un perfil de puntuaciones en cuatro subescalas que representan dimensiones de sintomatología: síntomas somáticos, ansiedad e insomnio, disfunción social y depresión. Cada una de estas dimensiones consta de 7 ítems, siendo un total de 28. Además, como medida global de severidad del malestar se usa la puntuación total del General Health Questionnaire (GHQ-28) (Goldberg \& Hillier, 1979; Lobo et al., 1986).

Cada ítem consiste en una pregunta que indaga si el individuo ha experimentado recientemente un síntoma o ítem de conducta particular, en una progresión que va desde menos de lo habitual a mucho más de lo habitual. En este estudio, se aplicó la puntuación tipo Likert con valores de 0 a 3 en las categorías de respuesta. El puntaje total se obtuvo mediante la sumatoria simple de las calificaciones en cada uno de los ítems.

\section{Procedimiento}

El programa de bienestar cuerpo-mente se llevó a cabo durante 12 semanas (Adeniyi et al., 2010). Consistió en dos clases a la semana, de 90 minutos de duración (Chambliss, 2005). De acuerdo al American College of Sports Medicine (American College of Sports Medicine, 1998; Durstine, Moore, Painter, \& Roberts, 2009), el ejercicio para personas con enfermedades crónicas debe tener una estructura en tres partes, que en este estudio fueron estructuradas de la siguiente manera.

Fase inicial (Bishop, 2003). Comenzaba con tres movimientos de QiGong. A continuación se llevaban a cabo 2 o 3 minutos de ejercicio aeróbico de intensidad moderada (tal como andar con o sin elevaciones de brazos simultáneas), seguidos de 2 o 3 minutos de ejercicios de expresión corporal y juegos. La duración del ejercicio aeróbico fue incrementándose gradualmente, de tal forma que al final de las 12 semanas los participantes realizaban 20 minutos de ejercicio aeróbico de intensidad ligera a moderada. 
Fase principal. Los siguientes 55 minutos de la clase fueron técnicas fisioterápicas con objetivos precisos para la estabilización o movilización articular (Kaltenborn, Kaltenborn, \& Vollowitz, 2009), para la reprogramación neuromotriz (Evjenth, 2001), para el estiramiento muscular (Tricás, Lucha, Hidalgo, \& Evjenth, 2012; TricásMoreno, Lucha-López, Lucha-López, SalaveraBordás, \& Vidal-Peracho, 2016) y para la movilización del sistema nervioso (Shacklock, 2005) que se iban variando de acuerdo al principal objetivo terapéutico de la sesión.

Fase final. Tenía una duración de 15 minutos e incluía, alternativamente, técnicas de relajación (Relajación Progresiva de Jacobson y Entrenamiento Autógeno de Schultz) y visualización de situaciones positivas y meditación (Hartmann et al., 2012).

La recogida de datos se llevó a cabo durante la semana previa al comienzo del programa y en la consecutiva a la finalización del mismo.

\section{Análisis}

Se analizaron los datos con el programa estadístico SPSS versión 16.0. Se calcularon estadísticos descriptivos de las características de los participantes y de las variables dependientes, la media y la desviación estándar para las variables cuantitativas y los porcentajes para las cualitativas.

La comparación de hipótesis en muestras relacionadas se realizó con la prueba de los rangos de Wilcoxon, a los valores de las variables dependientes en primera y segunda medición.

Se calculó el tamaño del efecto con el estadístico d de Cohen, para cuantificar la diferencia, de manera que se pudo interpretar mejor en términos de magnitud e importancia, para no confundir la significación estadística del resultado con su relevancia práctica (Cohen, 1992). Se utilizaron las siguientes categorías de interpretación del tamaño del efecto en función de los valores de la $\mathrm{d}$ de Cohen: $0.2-0.5$, pequeño; 0.6-0.8, mediano y más de 0.8, grande. Se realizó el estudio de la magnitud de la asociación entre variables cuantitativas con el coeficiente de correlación Rho de Spearman.

Se usaron test no paramétricos porque no se pudo asumir la distribución normal de las variables, y se estableció un nivel de significación del $5 \%$ (valor de $\mathrm{p}<0.05$ ) para rechazar la hipótesis nula.

\section{Resultados}

La Tabla 1 presenta las características básicas de la muestra. En la Tabla 2 se describen los valores medios de los factores de riesgo cardiovascular de la población de estudio y los de referencia en población general; el perímetro de cintura, la glucemia, la HbA1c, el fibrinógeno neto y la PCR US estaban elevados en la población de estudio.

\section{TABLA 1}

Características básicas de la muestra

\begin{tabular}{ll}
\hline Número muestral & $n=25$ \\
Mujeres & $n=20(80 \%)$ \\
Varones & $n=5(20 \%)$ \\
Edad (años) & $58 \pm 7.8$ \\
Edad inicio diabetes (años) & $47.3 \pm 10$ \\
Menores de 65 años & $n=21(84 \%)$ \\
Trabajadores en activo & $n=3(12 \%)$ \\
\hline
\end{tabular}

Fuente: elaboración propia.

\section{TABLA 2}

Descripción de los valores medios de los factores de riesgo cardiovascular de la muestra

\begin{tabular}{lll} 
& Valores en la muestra & Valores de referencia \\
\hline IMC & $34.8 \pm 6$ & $18.5-24.9^{*}$ \\
Perimetro de cintura (cm) & $105.3 \pm 12.3$ & $<88$ mujeres / 102 hombres ** \\
TAS (mmHg) & $133 \pm 10.8$ & $<140^{*}$ \\
TAD (mmHg) & $79.8 \pm 5.1$ & $<90^{*}$ \\
Colesterol total (mg/dl) & $172.7 \pm 20.1$ & $<200^{*}$ \\
HDL-c (mg/dl) & $58.4 \pm 10$ & $>50^{*}$ \\
LDL-c (mg/dl) & $92.1 \pm 16.4$ & $<130^{*}$ \\
Trigicéridos (mg/dl) & $122.2 \pm 88.3$ & $<200^{*}$ \\
Glucemia (mg/dl) & $136.9 \pm 35$ & $<100^{* * *}$ \\
HbAlc $(\%)$ & $6.6 \pm 1.2$ & $<5.7 \%^{* * *}$ \\
Fibrinogeno neto (mg/dl) & $404.7 \pm 77.3$ & $200-400^{* * * *}$ \\
PCR US (mg/L) & $4.9 \pm 6$ & $<3^{*}$ \\
\hline
\end{tabular}

Notas: *SEEDO 2007. **ATP III 2002.

***ADA 2012.****Gailani y Neff (2008).

Fuente: elaboración propia.

La distribución porcentual de los desórdenes somáticos coexistentes con la patología diabética y los factores de riesgo cardiovascular pueden ser consultados en la Figura 1. 


\section{Figura 1}

Distribución porcentual de los desórdenes somáticos coexistentes
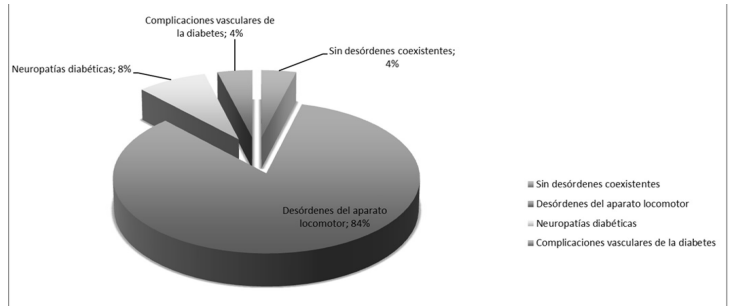

Fuente: elaboración propia.

En la Tabla 3 se pueden consultar los valores medios de las diferentes subescalas del GHQ, así como el valor medio de la suma de las mismas, antes y después de la intervención. Se muestran también los valores de $\mathrm{p}$, que fueron significativos para todas las variables y los de la d de Cohen. El tamaño del efecto de la intervención fue grande en las subescalas relacionadas con los síntomas somáticos y la disfunción social y en la puntuación total del test. Sin embargo, fue pequeño en las subescalas de ansiedad e insomnio y depresión severa.

\section{TABLA 3}

Descripción de los valores medios del test GHQ antes y después de la intervención y comparación de los mismos

\begin{tabular}{llllll}
\hline & \multicolumn{5}{c}{ Estadístic } \\
GHQ & Preintervenció & Postintervenció & \multicolumn{2}{c}{ o de } \\
Wilcoxon & Valor de $p$ & $d$ de Cohen \\
\hline Escala A: Síntomas Somáticos & $13.5 \pm 3.6$ & $9.3 \pm 2.7$ & -3.937 & $0^{*}$ & 1.3 \\
Escala B: Ansiedad Insomnio & $11.9 \pm 3.4$ & $10.2 \pm 3.3$ & -2.347 & $0.019^{*}$ & 0.5 \\
Escala C: Disfunción Social & $14.8 \pm 2.2$ & $12.2 \pm 2.8$ & -3.394 & $0.001^{*}$ & 1 \\
Escala D: Depresión Severa & $9.2 \pm 4.4$ & $7.8 \pm 3.2$ & -2.041 & $0.041^{*}$ & 0.4 \\
Puntuación total GHQ-28 & $48.7 \pm 10.7$ & $39.9 \pm 8.3$ & -3.824 & $0^{*}$ & 0.9 \\
\hline
\end{tabular}

Nota: ${ }^{*} p<0.05$. Categorías de interpretación del tamaño del efecto en función de los valores de la d de Cohen: 0.2-0.5, pequeño; 0.6-0.8: mediano; más de 0.8, grande. Fuente: elaboración propia.

La Tabla 4 muestra los resultados obtenidos por los registros de la VAS en su forma horizontal, obteniéndose una disminución muy importante de los valores tras la intervención con un tamaño del efecto grande.
TABLA 4

Descripción de los valores medios de la VAS antes y después de la intervención y comparación de los mismos

\begin{tabular}{llllll}
\hline & \multirow{2}{*}{ Preintervención } & Postintervención & Estadistico & de & \\
& & Wilcoxon & Valor de $p$ & $d$ de Cohen \\
\hline VAS & $6.1 \pm 2.5$ & $3.1 \pm 2.2$ & -4 & $0^{*}$ & 1.3 \\
\hline
\end{tabular}

Nota: $* p<0.05$. Categorías de interpretación del tamaño del efecto en función de los valores de la d de Cohen: 0.2-0.5, pequeño; 0.6-0.8: mediano; más de 0.8, grande. Fuente: elaboración propia.

Las relaciones existentes entre la variable dolor, medida con la VAS y el malestar psicológico cuantificado con el GHQ, se muestran en la Tabla 5 que incluye los valores del coeficiente de correlación entre estas variables y su significación estadística, antes y después de la intervención.

\section{TABLA 5}

Valores del coeficiente de correlación entre el GHQ y la VAS, y su significación estadística, antes y después de la intervención

\begin{tabular}{lllll}
\hline \multicolumn{3}{c}{ Preintervención } & \multicolumn{2}{l}{ Postintervención } \\
GHQ & VAS & Valor de $p$ & VAS & Valor de $p$ \\
\hline Escala A: Síntomas Somáticos & 0.433 & $0.044^{*}$ & 0.467 & $0.025^{*}$ \\
Escala B: Ansiedad e Insomnio & 0.513 & $0.009^{*}$ & 0.489 & $0.018^{*}$ \\
Escala C: Disfunción Social & 0.341 & 0.103 & 0.015 & 0.946 \\
Escala D: Depresión Severa & 0.218 & 0.307 & 0.388 & 0.101 \\
Puntuación total GHQ-28 & 0.474 & $0.03^{*}$ & 0.249 & 0.304 \\
\hline \multicolumn{5}{c}{ Nota: *p $p<0.05}$. \\
\end{tabular}

\section{Discusión}

En el presente estudio, se analizaron los datos correspondientes a una población de 25 pacientes diabéticos tipo 2 , con una proporción predominantemente femenina (80\%), al igual que en la población general (51\%) (OrozcoBeltrán et al., 2007), aunque con un porcentaje claramente superior.

En el momento inicial su edad media era de 58 años, con una evolución de la enfermedad de más de 10 años, con valores medios de hemoglobina glicosilada inferiores al $7 \%$, lo cual refleja un buen control de la enfermedad a pesar de la larga evolución de la misma (ADA, 2012). A pesar de la relativamente baja edad media, y que el $84 \%$ eran menores de 65 años, solo tres 
personas (12\%) eran trabajadores activos. Esta relación entre diabetes e incapacidad laboral, particularmente en pacientes obesos ya ha sido observada previamente por otros autores (Cawley, Rizzo, \& Haas, 2008) y se relaciona con un eventual descenso en la eficiencia que estas personas pueden sufrir debido a las alteraciones funcionales en algunos de sus órganos (Villiger, Straumann, \& Grimm, 1990). Se ha observado también, aunque en grupos muy concretos de población activa, que la obesidad por sí sola también incrementa el riesgo de incapacidad laboral debido a la osteoartritis y a la enfermedad cardiovascular (Claessen, Arndt, Drath, \& Brenner, 2009).

Nuestra muestra fue, además, una población con un alto riesgo cardiovascular, y la enfermedad cardiovascular se ha relacionado, así mismo, con incapacidad laboral (Mäntyniemi et al., 2012). En este caso, dicho riesgo estaba definido por varios factores: la diabetes de más de 10 años de evolución (ATP III, 2002); la obesidad con un predominio de localización central de la grasa, con un perímetro de cintura (105.3 \pm 12.3) por encima de los valores de referencia (ATP III, 2002) que es el morfotipo que define un mayor nivel de peligrosidad cardiovascular (Despres, Moorjani, Lupien, Tremblay, Nadeau, \& Bouchard, 1990) y los valores elevados de fibrinógeno neto $(404.7 \pm 77.3)$ (Gailani \& Neff, 2008) y de proteína C Reactiva Ultrasensible (4.9 \pm 6) (Sociedad Española de Diabetes, 2009) lo que indicaba un estado procoagulante y proinflamatorio que contribuía al riesgo cardiovascular (Dehghan et al., 2007).

Aún a pesar de todo ello, los valores dentro de los de referencia (Rubio et al., 2007) de la TAS $(133 \pm 10.8)$ y de la TAD $(79.8 \pm 5.1)$, así como los de los lípidos sanguíneos (Arancetaa et al., 2003); colesterol total $(172.7 \pm 20.1)$, HDL-c $(58.4 \pm 10)$, LDL-c $(92.1 \pm 16.4)$ y triglicéridos $(122.2 \pm 88.3)$ muestran que los pacientes tenían un control metabólico aceptable.

Sin embargo, la salud de estas personas se veía afectada negativamente también por otros desórdenes somáticos coexistentes. Hasta un $96 \%$ de la muestra tenía patologías que pueden provocar modificaciones en la percepción neural y/o aparición de síntomas como el dolor, con valores preintervención en la VAS de $6.1 \pm 2.5$. Los desórdenes del aparato locomotor presentes en un $84 \%$ de nuestra población eran la fuente principal de dolor. El aumento del peso y la localización de la grasa corporal podían contribuir a la aparición de síntomas musculoesqueléticos, dado que provocan aumentos de las desviaciones posturales y de las disfunciones que cursan con dolor y disminución del movimiento (Gilleard \& Smith, 2007; Hooper, Stellato, Hallowell, Seitz, \& Moskowitz, 2007), especialmente en columna vertebral, rodillas y pies (Fabris de Souza et al., 2005). Esta situación justificó el diseño de un programa de intervención específico para estos pacientes, que incluía ejercicios de fisioterapia con un alto potencial terapéutico en el tratamiento de su compleja situación clínica (Adeniyi et al., 2010; Evjenth, 2001; Kaltenborn et al., 2008; Lucha-López et al., 2012; Sartor et al., 2012; Tricas et al., 2012), dada además la relación existente entre las alteraciones funcionales y el menor bienestar psicológico (González et al., 2011; Tsenkova, Karlamangla, \& Ryff, 2016).

Los parámetros de salud de la población se describieron también en relación con los síntomas psicológicos. Los pacientes del estudio, en el momento inicial, puntuaron alto en las cuatro subescalas del GHQ, observándose síntomas de malestar psicológico especialmente en la de disfunción social $(14.8 \pm 2.2)$, seguida de la de síntomas somáticos $(13.5 \pm 3.6)$, ansiedad e insomnio $(11.9 \pm 3.4)$ y depresión severa $(9.2 \pm$ 4.4). La coexistencia de la diabetes con estados de ansiedad y de depresión ha sido previamente registrada en la bibliografía (Tovilla-Zárate et al., 2012). La falta de desempeño social (Gonzalez et al., 2011), el predominio de sexo femenino (Fisher, Chesla, Mullan, Skaff, \& Kanter, 2001), la obesidad y el riesgo cardiovascular (Khuwaja et al., 2010), la presencia de complicaciones (Gonzalez et al., 2011; Nouwen et al., 2011; Tovilla-Zárate et al., 2012), las limitaciones físicas (Gayman, Turner, \& Cui, 2008) y el dolor crónico (Bair, Robinson, Katon, \& Kroenke, 2003) son todos ellos factores que se han asociado 
en pacientes diabéticos tipo 2 con malestar psicológico y que pueden haber favorecido la alta puntuación obtenida en el GHQ en el presente estudio. Una de las últimas publicaciones al respecto (Tovilla-Zárate et al., 2012) señala, además, la necesidad de abordar los síntomas psicológicos en el tratamiento de la diabetes ya que, a su vez, perjudican el control de la glucemia y favorecen la evolución de la misma.

La revisión bibliográfica y los datos obtenidos del GHQ en el momento inicial aconsejaron dedicar una parte del programa de intervención a técnicas psicológicas para el tratamiento de los síntomas hallados, tales como meditación, relajación y visualización (Hartmann et al., 2012) y a ejercicios como el Qigong (Liu et al., 2010) que han dado muestras de mejorar el bienestar psíquico de estos pacientes.

El diseño del programa de estudio parece haber sido adecuado, dada la eficacia terapéutica observada para el dolor y el aumento del bienestar psicológico, con grandes efectos en la VAS, en la puntuación total del GHQ y en las subescalas de síntomas somáticos y de disfunción social, aunque en las subescalas de ansiedad e insomnio y de depresión se obtuviera solo pequeños efectos.

Nuestros resultados sugieren que no es posible estudiar el dolor y el malestar psicológico como fenómenos aislados. Recientemente, se ha observado como el dolor predecía, incluso, la efectividad del tratamiento farmacológico antidepresivo en pacientes diabéticos tipo 2 (Anderson, Gott, Sayuk, Freedland, \& Lustman, 2010).

En la muestra, tanto antes como después de la intervención, los pacientes con más dolor obtenían peores valores en la subescala de síntomas somáticos, en la de ansiedad y de insomnio. La posibilidad de que el aumento de los síntomas físicos influenciara la puntuación de la escala de síntomas somáticos del GHQ ya ha sido descrita en la literatura (Papassotiropoulos $\&$ Heun, 1999), por lo que en estos casos no sería un indicador válido de somatización, sino que sería más bien un reflejo de la importancia de los síntomas físicos.

La relación del dolor con la ansiedad y el insomnio se mantenía en nuestros pacientes incluso tras la intervención. La intensidad del dolor, a pesar de la gran eficacia terapéutica obtenida, se situó en niveles de dolor ligero a moderado (3.1 \pm 2.2) (Gómez-Batiste et al., 2002) y esta podría ser la razón por la que persistió la relación con la ansiedad y el insomnio y por lo que, en esta escala, se obtuvo un tamaño del efecto terapéutico pequeño, dado que altos niveles de dolor crónico son generadores de estrés emocional (Bair et al., 2003).

A pesar de lo positivo de todo lo expuesto, se ha de tener en cuenta que se puede establecer una serie de limitaciones al estudio. La selección de la muestra es una de ellas, dado que la adscripción al programa de entrenamiento fue voluntaria. Se ha visto que los participantes voluntarios en programas de educación diabética están altamente motivados y ello favorece la obtención de buenos resultados (García \& Morales, 2015; Schäfer et al., 2010). La asistencia voluntaria a programas de educación diabética también favorece la adherencia al tratamiento completo de la diabetes (QuirósMorales \& Villalobos-Pérez, 2007). La asociación existente entre las limitaciones físicas y el malestar psicológico hace difícil determinar la especificidad de la acción en la intervención fisioterápica y la psicoterápica.

Ahora bien, los esperanzadores resultados encontrados mediante esta intervención mixta sobre el entorno físico y el psicológico pueden servir de orientación para el desarrollo de futuros programas e investigaciones que también tengan en cuenta los factores concomitantes relacionados con los síntomas de malestar psíquico en el paciente diabético y que no restrinja su manejo al uso de fármacos.

En síntesis, se puede concluir, que el estudio se desarrolló en una población con una compleja situación clínica, que favorecía la escasa proporción de participación laboral activa observada, aún a pesar de que la mayoría eran menores de 65 años.

El programa de bienestar cuerpo-mente, adaptado a dicha situación, alivió el dolor de los pacientes, cuyo origen fueron las comorbilidades existentes. También, aumentó el bienestar psíquico, reduciendo los síntomas psicológicos en 
cada una de las subescalas examinadas, siendo el efecto terapéutico de notable intensidad para los síntomas somáticos y la disfunción social. Las mejoras en el dolor, tras la intervención, permiten que desaparezca la relación entre este y el malestar psicológico general, aunque persiste un nivel de dolor de ligero a moderado en relación con los síntomas somáticos y la ansiedad y el insomnio. El enfoque cuerpo-mente objeto de esta investigación, basado en técnicas especializadas fisioterápicas y psicológicas, ha mostrado ser una opción de elección para el manejo multidisciplinar en los pacientes diabéticos estudiados.

\section{Agradecimientos}

Ante todo, agradecer la inestimable participación de los pacientes y sus familias en el estudio, demostrando una inagotable paciencia y actitud positiva y un gran respeto y solidaridad para con la investigación relacionada con su enfermedad. También a las instituciones: al Servicio Aragonés de Salud, a la Facultad de Ciencias de la Salud de la Universidad de Zaragoza y a la Unidad de Investigación en Fisioterapia por el préstamo desinteresado de sus instalaciones e infraestructuras y por la amable colaboración de su personal.

\section{Referencias}

American Diabetes Association [ADA] (2012). Standards of medical care in diabetes 2012. Diabetes Care, 35(Suppl 1), 11-63.

Adeniyi, A. F., Fasanmade, A. A., Sanya, A. O., \& Borodo, M. (2010). Neuromusculoskeletal disorders in patients with type 2 diabetes mellitus: outcome of a twelve-week therapeutic exercise programme. Nigerian Journal of Clinical Practice, 13(4), 403-408.

American College of Sports Medicine. (1998). Exercise and physical activity for older adults: Position stand. Medicine and Science of Sports and Exercise Journal, 30, 992-1008.
Anderson, R. J., Gott, B. M., Sayuk, G. S., Freedland, K. E., \& Lustman, P. J. (2010). Antidepressant pharmacotherapy in adults with type 2 diabetes: Rates and predictors of initial response. Diabetes Care, 33(3), 485-489.

Arancetaa, J., Foza, M., Gilc, B., Joverd, E., Mantillae, T., Millánd, J. ... Moreno, B. (2003). Documento de consenso: obesidad y riesgo cardiovascular. Clinica e Investigación en Arteriosclerosis, 15(5), 196-233.

Bair, M. J., Robinson, R. L., Katon, W., \& Kroenke, K. (2003). Depression and pain comorbidity: A literature review. Archives of Internal Medicine, 163(20), 2433-2445.

Bishop, D. (2003). Warm up II: Performance changes following active warm up and how to structure the warm up. Sports Medicine, 33(7), 483-498.

Buchberger, B., Huppertz, H., Krabbe, L., Lux, B., Mattivi, J. T., \& Siafarikas, A. (2016). Symptoms of depression and anxiety in youth with type 1 diabetes: A systematic review and meta-analysis. Psychoneuroendocrinology, 70(1), 70-84.

Cawley, J., Rizzo, J. A., \& Haas, K. (2008). The association of diabetes with job absenteeism costs among obese and morbidly obese workers. Journal of Occupational and Environmental Medicine, 50(5), 527-534.

Chambliss, H. O. (2005). Exercise duration and intensity in a weight-loss program. Clinical Journal of Sport Medicine, 15(2), 113-115.

Chien, I. -C., \& Lin, C. -H. (2016). Increased risk of diabetes in patientes with anxiety disorders: A population-based study. Journal of Psychosomatic Research, 86(1), 47-52.

Claessen, H., Arndt, V., Drath, C., \& Brenner, H. (2009). Overweight, obesity and risk of work disability: A cohort study of construction workers in Germany. Occupational and Environmental Medicine, 66(6), 402-409.

Cohen, J. (1992). A power primer. Psychology Bulletin, 112, 155-159. 
Dehghan, A., Kardys, I., de Maat, M. P., Uitterlinden, A. G., Sijbrands, E. J., Bootsma, A. H., ... Witteman, J. C. (2007). Genetic variation, C-reactive protein levels, and incidence of diabetes. Diabetes, 56(3), 872-878.

Despres, J. P., Moorjani, S., Lupien, P. J., Tremblay, A., Nadeau, A., \& Bouchard, C. (1990). Regional distribution of body fat, plasma lipoproteins, and cardiovascular disease. Arteriosclerosis, 10, 497-511.

Durstine, J. L., Moore, G., Painter, P., \& Roberts, S. (2009). ACSM's exercise management for persons with chronic disease and disabilities (3.a ed. ). Illinois: American College of Sports Medicine, Human Kinetics.

Evjenth, O. (2001). Advanced level course in OMT: Kaltenborn-Evjenth concept. Zaragoza: Health Sciences Faculty, Zaragoza University, Servicio de Publicaciones.

Fabris de Souza, S. A., Faintuch, J., Valezi, A. C., Sant'Anna, A. F., Gama-Rodrigues, J. J., de Batista Fonseca, I. C., \& de Melo, R. D. (2005). Postural changes in morbidly obese patients. Obese Surgery, 15(7), 1013-1016.

Fisher, L., Chesla, C. A., Mullan, J. T., Skaff, M. M., \& Kanter, R. A. (2001). Contributors to depression in Latino and EuropeanAmerican patients with type 2 diabetes. Diabetes Care, 24(10), 1751-1757.

Fisher, L., Mullan, J. T., Arean, P., Glasgow, R. E., Hessler, D., \& Masharani, U. (2010). Diabetes distress but not clinical depression or depressive symptoms is associated with glycemic control in both cross-sectional and longitudinal analyses. Diabetes Care, $33,23-28$.

Gailani, D., \& Neff, A. T. (2008). Rare coagulation factor deficiencies. En R. Hoffman, E. J. Benz, Jr. \& S. J. Shattil (Eds.), Hoffman hematology: Basic principles and practice (5.a ed., Cap. 127). Philadelphia: Churchill Livingstone Elsevier.

García, I., \& Morales, I. V. (2015). Eficacia de la entrevista motivacional para promover la adherencia terapéutica en pacientes con diabetes mellitus tipo 2. Universitas Psychologica, 14(2), 511-522.
Gayman, M. D., Turner, R. J., \& Cui, M. (2008). Physical limitations and depressive symptoms: Exploring the nature of the association. Journals of Gerontology Series B: Psychological Sciences and Social Sciences, 63, 219-228.

Gilleard, W., \& Smith, T. (2007). Effect of obesity on posture and hip joint moments during a standing task, and trunk forward flexion motion. International Journal of Obesity, $31(2), 267-271$.

Goldberg, D. P., \& Hillier, V. F. (1979). A scaled version of the General Health Questionnaire. Psychological Medicine, 9, 139-145.

Goldberg, D. P., \& Williams, P. (1988). A user's guide to the General Health Questionnaire. United Kingdom: NFER-NELSON.

Gómez-Batiste, X., Madrid, F., Moreno, F., Gracia, A., Trelis, J., Nabal, M., ... Camell, H. (2002). Breakthrough cancer pain: Prevalence and characteristics in patients in Catalonia, Spain. Journal of Pain and Symptoms Management, 24(1), 45-52.

Gonzalez, J. S., Fisher, L., \& Polonsky, W. H. (2011). Depression in diabetes: Have we been missing something important? Diabetes Care, 34(1), 236-239. doi: 10.2337/dc10-1970

Hartmann, M., Kopf, S., Kircher, C., Faude-Lang, V., Djuric, Z., Augstein, F., ... Nawroth, P. P. (2012). Sustained effects of a mindfulnessbased stress-reduction intervention in type 2 diabetic patients: Design and first results of a randomized controlled trial (the Heidelberger Diabetes and Stress-study). Diabetes Care, 35(5), 945-947.

Hooper, M. M., Stellato, T. A., Hallowell, P. T., Seitz, B. A., \& Moskowitz, R. W. (2007). Musculoskeletal findings in obese subjects before and after weight loss following bariatric surgery. International Journal Obesity, 31 (1), 114-120.

Kaltenborn, F., Kaltenborn, T., \& Vollowitz, E. (2009). Manipulación-tracción de las extremidades y columna. Técnicas básicas de thrust. Fisioterapia manual. Evaluación 
y tratamiento articular básicos (Vol. 3). Zaragoza: OMT España.

Khuwaja, A. K., Lalani, S., Dhanani, R., Azam, I. S., Rafique, G., \& White, F. (2010). Anxiety and depression among outpatients with type 2 diabetes: A multi-centre study of prevalence and associated factors. Diabetology $\mathbb{E}$ Metabolic Syndrome, 2(72), 1-10. doi:10.1186/1758-5996-2-72

Liu, X., Miller, Y. D., Burton, N. W., \& Brown, W. J. (2010). A preliminary study of the effects of Tai Chi and Qigong medical exercise on indicators of metabolic syndrome, glycaemic control, health-related quality of life, and psychological health in adults with elevated blood glucose. British Journal Sports Medicine, 44(10), 704-709.

Lobo, A., Pérez-Echeverría, M. J., \& Artal, J. (1986). Validity of the scaled version of the General Health Questionnaire (GHQ-28) in a Spanish population. Psychological Medicine, 16(1), 135-140.

Lobo, A., Pérez-Echeverría, M. J., JiménezAznárez, A., \& Sancho, M. A. (1988). Emotional disturbances in endocrine patients. Validity of the scaled version of the General Health Questionnaire (GHQ-28). British Journal of Psychiatry, 152, 807-812.

Lucha-López, M. O., Lucha-López, A. C., Vidal-Peracho, C., Tricás-Moreno, J. M., Estebanez-De Miguel, E., Salavera-Bordás, C., ... Caudevilla-Polo, S. (2012). Impact of supervised physiotherapeutic exercises for obese adults with diabetes mellitus type 2 . Journal of Physical Therapy Science, 24(12), 1299-1305.

Lucha-López, M. O., Lucha-López, A. C., Vidal-Peracho, C., Tricás-Moreno, J. M., Estébanez-De Miguel, E., Salavera-Bordás, C., \& Hidalgo-García, C. (2014). Analysis of a sample of type 2 diabetic patients with obesity or overweight and at cardiovascular risk: A cross sectional study in Spain. BMC Research Notes, 7. doi:10.1186/1756-0500-7-48

Mäntyniemi, A., Oksanen, T., Salo, P., Virtanen, M., Sjösten, N., Pentti, J., ... \& Vahtera, J. (2012). Job strain and the risk of disability pension due to musculoskeletal disorders, depression or coronary heart disease: A prospective cohort study of 69,842 employees. Occupational and Environmental Medicine, 69(8), 574-581.

Merkes, M. (2010). Mindfulness-based stress reduction for people with chronic diseases. Australian Journal of Primary Health, 16(3), 200-210.

Nicolucci, A., Balducci, S., Cardelli, P., Cavallo, S., Fallucca, S., Bazuro, A., ... Pugliese, G. (2012). Italian diabetes exercise study investigators. Relationship of exercise volume to improvements of quality of life with supervised exercise training in patients with type 2 diabetes in a randomized controlled trial: The Italian Diabetes and Exercise Study (IDES). Diabetologia, 55(3), 579-588.

Norton, K., Whittingham, N., Carter, L., Kerr, D., Gore, C., \& Marfell-Jones, M. (1996). Measurement techniques in anthropometry. En K. Norton \& T. Olds (Eds.), Anthropometrica (pp. 25-75). Syndey: University of New South Wales Press.

Nouwen, A., Nefs, G., Caramlau, I., Connock, M., Winkley, K., Lloyd, C. E., ... Pouwer, F. (2011). European depression in diabetes research consortium. Prevalence of depression in individuals with impaired glucose metabolism or undiagnosed diabetes: A systematic review and metaanalysis of the European Depression in Diabetes (EDID) Research Consortium. Diabetes Care, 34(3), 752-762.

Orozco-Beltrán, D., Gil-Guillen, V. F., Quirce, F., Navarro-Perez, J., Pineda, M., Gomez-dela-Cámara, A., ... Serrano-Rios, M. (2007). Collaborative diabetes study investigators. Control of diabetes and cardiovascular risk factors in patients with type 2 diabetes in primary care. The gap between guidelines and reality in Spain. International Journal of Clinical Practice, 61 (6), 909-915.

Papassotiropoulos, A., \& Heun, R. (1999). Screening for depression in the elderly: A study on misclassification by 
screening instruments and improvement of scale performance. Progress in NeuroPsychopharmacology $\mathbb{E}$ Biological Psychiatry, 23(3), 431-446.

Pariser, G., DeMeuro, M. A., Gillette, P., \& Stephen, W. (2010). Outcomes of an education and exercise program for adults with type 2 diabetes, and comorbidities that limit their mobility. Cardiopulmonary Physical Therapy Journal, 21 (2), 5-12.

Quirós-Morales, D., \& Villalobos-Pérez, A. (2007). Comparación de factores vinculados a la adherencia al tratamiento en diabetes mellitus tipo II entre una muestra urbana y otra rural de Costa Rica. Universitas Psychologica, 6(3), 679-688.

Rubio, M. A., Salas-Salvadó, J., Barbany, M., Moreno, B., Aranceta, J., Bellido, D., ... Vidal J. (2007). Consenso SEEDO 2007 para la evaluación del sobrepeso y la obesidad y el establecimiento de criterios de intervención terapéutica. Revista Española de Obesidad, 112(15), 7-48.

Sartor, C. D., Watari, R., Pássaro, A. C., Picon, A. P., Hasue, R. H., \& Sacco, I. C. (2012). Effects of a combined strengthening, stretching and functional training program versus usual-care on gait biomechanics and foot function for diabetic neuropathy: A randomized controlled trial. BMC Musculoskeletal Disorders, 13(36), 1-10. doi: 10.1186/1471-2474-13-36

Schäfer, I., Küver, C., Gedrose, B., von Leitner, E. C., Treszl, A., Wegscheider, K., ... Kaduszkiewicz, H. (2010). Selection effects may account for better outcomes of the German Disease Management Program for type 2 diabetes. BMC Health Services Research, 10(351), 1-10. doi:10.1186/1472-6963-10-351

The Seventh Report of the Joint National Committee on Prevention, Detection, Evaluation, and Treatment of High Blood Pressure. (2004). National High Blood Pressure Education Program (NIH Publication, 04-5230). Washington, DC: Department of Health and Human
Services, National Institutes of Health. National Heart, Lung, and Blood Institute. Shacklock, M. (2005). Clinical neurodynamics: A new system of musculoskeletal treatment. Oxford: Elsevier.

Sociedad Española de Diabetes. (2009). Diabetes mellitus y riesgo cardiovascular. Recomendaciones del Grupo de Trabajo Diabetes Mellitus y Enfermedad Cardiovascular de la Sociedad Española de Diabetes [Documento de consenso]. Avances en Diabetología, 25, 449-454.

Telli, O., \& Cavlak, U. (2006). Measuring the pain threshold and tolerance using electrical stimulation in patients with Type II diabetes mellitus. Journal of Diabetes and its Complications, 20(5), 308-316.

Third Report of the National Cholesterol Education Program. (2002). Expert panel on detection, evaluation, and treatment of high blood cholesterol in adults (Adult Treatment Panel III, final report). Circulation, 106, 3143-3421.

Tovilla-Zárate, C., Juárez-Rojo, I., Peralta Jimenez, Y., Jimenez, M. A., Vazquez, S., Bermudez-Ocaña, D., ... Narvaez, L. L. (2012). Prevalence of anxiety and depression among outpatients with type 2 diabetes in the Mexican population. PLoS One, 7(5), e36887. doi:10.1371/ journal.pone.0036887

Trento, M., Raballo, M., Trevisan, M., Sicuro, J., Passera, P., Cirio, L., ... Porta, M. (2012). A cross-sectional survey of depression, anxiety, and cognitive function in patients with type 2 diabetes. Acta Diabetologica, 49(3), 199-203.

Tricás, J. M., Lucha, O., Evjenth, O., \& Hidalgo, C. (2012). Estiramiento y autoestiramiento muscular en terapia manual ortopédica. Zaragoza: Asociación Española de Terapia Manual Ortopédica.

Tricás-Moreno, J. M., Lucha-López, O., LuchaLópez, A., Salavera-Bordás, C., \& VidalPeracho, C. (2016). Optimizing physical therapy for ankylosing spondylitis: A case study in a young football player. Journal of Physical Therapy Science, 28(4), 1392-1397. 
Tsenkova, V. K., Karlamangla, A. S., \& Ryff, C. D. (2016). Parental history of diabetes, positive affect, and diabetes risk in adults: Findings from MIDUS. Annals of Behavioral Medicine, 50(6), 836-843.

Usán, P., Salavera, C., Murillo, V., \& Mejías, J.J. (2016). Relación entre motivación, compromiso y autoconcepto en adolescentes: estudio con futbolistas. Cuadernos de Psicología del Deporte, 16(1), 199-209.

Vidal-Peracho, C., Lucha-López, M., LuchaLópez, A., Tricás-Moreno, J., EstébanezDe Miguel, E., \& Bernués-Vázquez, L. (2014). A descriptive study of health status and health related quality of life in selected outpatients with type 2 diabetes, pathological body mass index and cardiovascular risk in Spain. Diabetology $\mathfrak{E}$ Metabolic Syndrome, 6(1), 135.

Vileikyte, L., Peyrot, M., Gonzalez, J. S., Rubin, R. R., Garrow, A. P., Stickings, D., ... Boulton, A. J. (2009). Predictors of depressive symptoms in persons with diabetic peripheral neuropathy: A longitudinal study. Diabetologia, 52, 1265-1273.

Villiger, U., Straumann, M., \& Grimm, J. J. (1990). Social and economic aspects in diabetes mellitus. Schweizerische Rundschau Für Medizin Praxis, 79(41), 1217-1220.

\section{Notas}

* Artículo de investigación. 